\title{
ON VARIATIONAL PRINCIPLES OF CONFORMAL MAPPINGS
}

\author{
V. N. DUBININ AND E. G. PRILEPKINA \\ Dedicated to the 100th anniversary of Gennadiǔ Mikhailovich Goluzin's birth
}

\begin{abstract}
Refinements and generalizations of the classical variational principles of conformal mappings are presented; mainly, they follow from potential theory and symmetrization. Part of the results can be viewed as properties of Robin functions and Robin capacities, and also as distortion theorems for univalent functions in finitely connected domains.
\end{abstract}

\section{§1. INTRODUCTION AND MAIN DEFINITIONS}

The principles mentioned in the title are of major importance in the theory of functions of a complex variable and in the mechanics of solids [1, 2]. In the present paper, we are concerned with qualitative variational principles, i.e., statements enabling us to judge the variation of a conformal map by the variation of the boundary of a domain. For instance, a classical qualitative variational principle says that if a function $w=f(z)$ maps a domain $D, \infty \in D \subset \Delta_{z}:=\{z:|z|>1\}$, conformally and univalently onto the exterior $\Delta_{w}$ of the unit disk in such a way that $f(\infty)=\infty$, then $\left|f^{\prime}(\infty)\right| \leq 1$, at an arbitrary point $z \in(\partial D) \cap\left(\partial \Delta_{z}\right)$ we have $\left|f^{\prime}(z)\right| \leq 1$ (if the derivative exists), and for every $\rho>1$ the level curve $|f(z)|=\rho$ is included in $|z| \geq \rho$. In other words, when $\Delta_{z}$ is deformed to $D$, the modulus of the derivative decays at infinity and at the fixed points on the boundary, and the level curves expand. Similar principles are valid for functions realizing conformal mapping onto other types of standard domains: the half-plane $H_{w}:=\{w: \operatorname{Im} w>0\}$ and the strip $S_{w}:=\{w: 0<\operatorname{Im} w<1\}$; see [2, Subsection 61] (in contrast to [2, $f$ is compared with the identity mapping; this does not lead to any loss of generality). The principles mentioned above can be regarded as properties of the complex potentials of stationary parallel plane vector fields. They follow, for example, from the Schwarz lemma or the maximum principle for harmonic functions. Various extensions and refinements of these principles have been known for some time. Moreover, modern methods of the geometric theory of functions of a complex variable (see 3]) make it possible to obtain new variational theorems that take into account additional normalization of a mapping or the nature of the deformation of the domain in question. However, these facts have not been reflected in the literature in due form. Our purpose is to fill this gap, at least partly. In the paper, we present variational principles that are derived from potential theory and symmetrization in a uniform way. For a canonical domain $K_{z}$, we study the properties of a map $f: D \rightarrow K_{w}$ in their dependence on the deformation $\left(D \backslash K_{z}\right) \cup\left(K_{z} \backslash D\right)$. If $D \subset K_{z}$, we use the term inner deformation, and if $D \supset K_{z}$, we use the term outer

2000 Mathematics Subject Classification. Primary 30C70, 30C85.

Key words and phrases. Variational principles, conformal mapping, distortion theorems, univalent functions, Robin function, Robin capacity, the capacity of a condenser, majorant domains, symmetrization, polarization.

This research was supported by the "Leading research school" program (grant no. Sh-9004.2006.1), by RFBR (grant no. 05-01-00099), and by FEB RAS (grant no. 06-III-A-01-013). 
deformation (of $K_{z}$ ). Principal attention will be paid to the variation of the modulus of the derivative of $f$, i.e., to dilation estimates. Along with the canonical domains mentioned above, we introduce also the annulus $K_{w}(R)=\{w: 1<|w|<R\}$ and the quadrangle $Q_{w}\left(a_{1}, a_{2}, a_{3}, a_{4}\right)$ (in the plane $\left.\mathbf{C}_{w}\right)$ with rectilinear edges and with vertices at the points $a_{1}, a_{2}, a_{3}, a_{4}$ (the enumeration agrees with the positive direction on the boundary). In accordance with the variational principles under study, we introduce the following classes of functions.

$\mathcal{V}_{1}$ is the class of functions $w=f(z)$ that map a domain $D=D(f), \infty \in D \subset \overline{\mathbf{C}}_{z}$, univalently and conformally onto the exterior of the unit disk $\Delta_{w}$ and admit an expansion of the form

near infinity.

$$
f(z)=\alpha z+O(1), \quad z \rightarrow \infty
$$

$\mathcal{V}_{2}$ is the class of functions $w=f(z)$ that map a domain $D=D(f)$ for which $\left(H_{z} \backslash D\right) \cup$ $\left(D \backslash H_{z}\right)$ is a bounded set onto the half-plane $H_{w}$ in a univalent and conformal way, and have an expansion of the form

$$
f(z)=z+O(1), \quad z \in D, \quad z \rightarrow \infty .
$$

$\mathcal{V}_{3}$ is the class of functions $w=f(z)$ that map a domain $D=D(f)$ with $\left(S_{z} \backslash D\right) \cup$ $\left(D \backslash S_{z}\right)$ bounded onto the strip $S_{w}$ in a conformal and univalent way and have the property

$$
\lim _{\substack{z \in+\infty \\ z \in D}} f(z)=+\infty, \quad \lim _{z \rightarrow-\infty} f(z)=-\infty .
$$

$\mathcal{V}_{4}(l)$ is the class of functions $w=f(z)$ satisfying the following conditions: the domain of $f$ is a quadrangle $D=D(f)$ with vertices $a_{1}, a_{2}, a_{3}, a_{4}$ enumerated in agreement with the positive direction on the boundary; the side $a_{1} a_{2}$ lies on the segment $[l, l+i], l>0$, and the side $a_{3} a_{4}$ lies on the segment $[i, 0] ; f$ maps $D$ conformally and univalently on the rectilinear quadrangle $Q(L, L+i, i, 0), L=L(f)>0$, with the following correspondence of vertices: $f\left(a_{1}\right)=L, f\left(a_{2}\right)=L+i, f\left(a_{3}\right)=i, f\left(a_{4}\right)=0$.

$\mathcal{V}_{5}$ is the class of functions $w=f(z)$ that are defined in a doubly-connected domain $D=D(f)$ separating the disk $|z| \leq 1$ from infinity, and map $D(f)$ conformally and univalently onto the annulus $K_{w}(R)$ in such a way that the outer boundary component of $D$ corresponds to the circle $|w|=R, R=R(f)$.

In the case of inner deformations, the functions in the classes indicated above obey some restrictions worthy of notice. For instance, if $D(f) \subset \Delta_{z}$ for some $f \in \mathcal{V}_{1}$, then the Schwarz lemma implies $|\alpha| \leq 1$, and equality may occur only if $f(z)=e^{i \varphi} z$ for some real $\varphi$. Thus, if $|\alpha|>1$, then the subclass $\left\{f \in \mathcal{V}_{1}: f^{\prime}(\infty)=\alpha, D(f) \subset \Delta_{z}\right\}$ is empty; if $|\alpha|=1$, this subclass is the collection of rotations about the origin. Next, if a function $f(z)=z+c / z+\cdots$ belongs to $\mathcal{V}_{2}$ and $D(f) \subset H_{z}$, then necessarily $c \geq 0$, and moreover, $c=0$ only for the function $f(z) \equiv z$. Comparison of the conformal moduli of quadrangles and annuli implies the following statements: in $\mathcal{V}_{3}$, inner deformations (i.e., such that $\left.D(f) \subset S_{z}\right)$ are possible only if

$$
\lim _{\substack{z \rightarrow+\infty \\ z \in D}}(f(z)-z)-\lim _{\substack{z \rightarrow-\infty \\ z \in D}}(f(z)-z) \geq 0
$$

the conditions $f \in \mathcal{V}_{4}(l)$ and $D(f) \subset Q_{z}(l, l+i, i, 0)$ imply $l \leq L$; finally, if $f \in \mathcal{V}_{5}$, then $D(f) \subset K_{z}(R)$ and necessarily $R(f) \leq R$.

In $\S 2$ of the present paper, we consider quadratic forms that depend on Robin functions (see 4]), and prove a fairly general variational theorem about the behavior of these forms under deformation of the domain in question. Many classical and modern statements of function theory are consequences of that theorem; among them we can name, in particular, the variational principles pertaining to dilation estimates for the function 
classes introduced above. In the same section, the main theorem is supplemented with certain statements of a special nature. In $\S 3$, we study variation of dilations under a given shift of points, the deformation of the domain being fixed. We compare inner and outer deformations in their impact on the nature of the conformal map. The final $\S 4$ is of an expository nature. In that section, we collect certain statements about the behavior of level curves under mappings in the above classes; these statements strengthen some well-known theorems; see [2]. In passing, we demonstrate the advantages of the symmetrization method in the proofs of such statements. The results of the paper can be viewed as supplements to the variational principles (see 2]) and to the method of majorizing domains (see [6]); both the former and the latter have found applications in various topics of mathematical physics. On the other hand, the theorems of $\S \S 2,3$ are applications of (and express new properties of) Robin functions and Robin capacities (see [7), which have recently gained considerable attention from researchers (see [7]-[13]). In particular, to a certain extent, the theorems of $\S 3$ answer a question by Duren (see [7, p. 188]) concerning the prospects of the use of the symmetrization method in the study of Robin capacities. In contrast to [7, we consider not only Robin functions with a pole at a point of a domain $B$, but also their analogs with a pole at a boundary point (see [14). We recall the definition.

Let $B$ be a domain whose boundary consists of a finite number of analytic Jordan curves, and let $\Gamma$ be a nonempty subset of $\partial B$ that is a finite union of nondegenerate arcs. Let $z_{0}$ be a finite point in $\bar{B} \backslash \Gamma$. We denote by $g(z)=g_{B}\left(z, z_{0}, \Gamma\right)$ the real continuous function of $\bar{B} \backslash\left\{z_{0}\right\}$ that is continuously differentiable on $\bar{B} \backslash\left(\Gamma \cup\left\{z_{0}\right\}\right)$ and harmonic in $B \backslash\left\{z_{0}\right\}$ and satisfies the following conditions:

$$
\begin{aligned}
g(z)=0 & \text { for } z \in \Gamma, \\
\frac{\partial g}{\partial n}(z)=0 & \text { for } z \in(\partial B) \backslash\left(\Gamma \cup\left\{z_{0}\right\}\right),
\end{aligned}
$$

and the function $g(z)+\log \left|z-z_{0}\right|$ is harmonic near $z_{0}$ (here $\partial / \partial n$ stands for differentiation along the inward normal to $\partial B$ ).

If $z_{0}=\infty$, the function $g_{B}\left(z, z_{0}, \Gamma\right)$ is defined similarly, with the only difference that $g_{B}\left(z, z_{0}, \Gamma\right)-\log |z|$ must be harmonic near infinity. For finitely connected domains with nonsmooth boundary, $g_{B}\left(z, z_{0}, \Gamma\right)$ is defined via conformal mapping (see [14]). For $z_{0} \in B$, the function $g_{B}\left(z, z_{0}, \Gamma\right)$ is called the Robin function of $B$ with pole at $z_{0}$. If $\Gamma=\partial B$, the Robin function coincides with the Green function. Also, we introduce the notation

$$
r\left(B, \Gamma, z_{0}\right)=\exp \left\{\lim _{z \rightarrow z_{0}}\left[g_{B}\left(z, z_{0}, \Gamma\right)+\log \left|z-z_{0}\right|\right]\right\}
$$

if $z_{0}$ is a finite point, and

$$
r(B, \Gamma, \infty)=\exp \left\{\lim _{z \rightarrow \infty}\left[g_{B}(z, \infty, \Gamma)-\log |z|\right]\right\} .
$$

If $z_{0}=\infty$ and $z_{0} \in B$, the quantity $r^{-1}\left(B, \Gamma, z_{0}\right)$ is called the Robin capacity of the set $\Gamma$ with respect to $B$. In the case where $B$ is simply connected, $\Gamma$ is an arc on the boundary of $B$, and $z_{0} \in(\partial B) \backslash \Gamma$, the quantity $r\left(B, \Gamma, z_{0}\right)$ was treated in essence in [15, 16]. The study of differences and ratios of Robin capacities is of particular interest; see [7, 11] and [17, Chapter 7.2].

The main tool in the proof of the theorems in $\S \S 2-4$ is the notion of the capacity of a generalized condenser; see 14] (we omit the word "generalized" in the sequel). Let $B$ be a finitely connected domain on the complex sphere $\overline{\mathbf{C}}_{z}$, and let $\bar{B}$ denote the compactification of $B$ via Carathéodory prime ends. We denote by $\partial B$ the boundary of the set of prime ends. By a neighborhood we mean an arbitrary open subset of $\bar{B}$. In what follows, if this does not lead to confusion, we identify the element of $\bar{B}$ corresponding 
to an interior point of $B$ with this point itself, and we denote by the same letter an accessible boundary point and its support. A condenser in $B$ is a triple $C=(B, \mathcal{E}, \Delta)$, where $\mathcal{E}=\left\{E_{k}\right\}_{k=1}^{n}$ is a collection of mutually disjoint and closed subsets of $\bar{B}$, and $\Delta=\left\{\delta_{k}\right\}_{k=1}^{n}$ is a collection of real numbers. For every $k, 1 \leq k \leq n$, the set $E_{k}$ will be called a plate of the condenser $C$, and $\delta_{k}$ will be called the level of potential (the potential, for short) of $E_{k}$. The capacity cap $C$ of the condenser $C$ is defined to be the greatest lower bound of the Dirichlet integrals

$$
I(v, B):=\iint_{B}|\nabla v|^{2} d x d y
$$

over all admissible functions $v(z)(z=x+i y)$, i.e., real-valued functions $v(z)$ continuous in $\bar{B}$, satisfying a Lipschitz condition near every finite point of $B$, and equal to $\delta_{k}$ in a neighborhood of $E_{k}, k=1, \ldots, n$. If the boundary of $\bigcup_{k=1}^{n} E_{k}$ consists of a finite number of nondegenerate connected components, and the portions $(\partial B) \backslash \bigcup_{k=1}^{n} E_{k}$ of the boundary are smooth, then there exists a unique function $u$ continuous in $\bar{B}$, harmonic in $B \backslash \bigcup_{k=1}^{n} E_{k}$, equal to $\delta_{k}$ on $E_{k}, k=1, \ldots, n$, and satisfying $\partial u / \partial n=0$ on $(\partial B) \backslash \bigcup_{k=1}^{n} E_{k}$. This function is called the potential function for $C$. By the Dirichlet principle, we have

$$
\operatorname{cap} C=I(u, B) \text {. }
$$

Also, we shall need the asymptotics of a condenser's capacity in a particular case treated in [14]. For a finite point $z_{0}$ of the complex sphere $\overline{\mathbf{C}}$, we denote by $D\left(z_{0}, r\right)$ the closed disk centered at $z_{0}$ and of radius $r>0$. For the point at infinity, we put $D(\infty, r):=\{z:|z| \geq 1 / r\}$. A closed set $\widetilde{D}\left(z_{0}, r\right), z_{0} \in \overline{\mathbf{C}}$, depending on a parameter $r>0$, will be called a near-disk centered at $z_{0}$ and of radius $r$ if for some $\varepsilon>0$ there exist positive functions $r_{j}(r), 0<r<\varepsilon, j=1,2$, such that

$$
D\left(z_{0}, r_{1}(r)\right) \subset \widetilde{D}\left(z_{0}, r\right) \subset D\left(z_{0}, r_{2}(r)\right)
$$

and $r_{j}(r) \sim r$ as $r \rightarrow 0, j=1,2$. Throughout, we use the notation $E\left(z_{0}, r, B\right)=$ $\widetilde{D}\left(z_{0}, r\right) \cap \bar{B}$ for a given domain $B \subset \overline{\mathbf{C}}$ and a given $z_{0} \in \bar{B}$. Let $B$ be a finitely connected domain without isolated boundary points. A point $z_{0} \in \bar{B}$ is said to be admissible if it belongs either to $B$ or to the interior of a smooth boundary arc of $B$ and, moreover, the derivative $\varphi^{\prime}$ exists at $z_{0}$ for every function $\varphi$ that maps $B$ conformally and univalently onto a Jordan domain bounded by a finite number of analytic curves. (The set of admissible points may be enlarged substantially by inclusion of, say, corner points; however, this falls beyond the scope of this paper.) Let $\Gamma$ be a closed subset of $\partial B$ consisting of finitely many nondegenerate connected components, let $Z=\left\{z_{k}\right\}_{k=1}^{n}$ be a collection of different admissible points of $\bar{B} \backslash \Gamma$, let $\Delta=\left\{\delta_{k}\right\}_{k=1}^{n}$ be a collection of reals with $\sum_{k=1}^{n} \delta_{k}^{2} \neq 0$, and let $\Psi=\left\{\psi_{k}\right\}_{k=1}^{n}$, where $\psi_{k}=\psi_{k}(r) \equiv \mu_{k} r$, and the $\mu_{k}(k=1, \ldots, n)$ are arbitrary positive numbers. For $r$ sufficiently small, we put $C(r ; B, \Gamma, Z, \Delta, \Psi):=\left(B,\left\{\Gamma, E\left(z_{1}, \psi_{1}(r), B\right), \ldots, E\left(z_{n}, \psi_{n}(r), B\right)\right\},\left\{0, \delta_{1}, \ldots, \delta_{n}\right\}\right)$. Theorem 7 in 14 yields the asymptotic formula

$$
\begin{aligned}
\operatorname{cap} C & (r ; B, \Gamma, Z, \Delta, \Psi) \\
= & \pi\left[\sum_{k=1}^{n} \alpha_{k} \delta_{k}^{2}\right]\left(-\frac{1}{\log r}\right) \\
& -\pi\left[\sum_{k=1}^{n} \alpha_{k} \delta_{k}^{2} \log \frac{r\left(B, \Gamma, z_{k}\right)}{\mu_{k}}+\sum_{k=1}^{n} \sum_{l=1, l \neq k}^{n} \alpha_{k} \delta_{k} \delta_{l} g_{B}\left(z_{k}, z_{l}, \Gamma\right)\right]\left(\frac{1}{\log r}\right)^{2} \\
& +o\left(\left(\frac{1}{\log r}\right)^{2}\right), \quad r \rightarrow 0,
\end{aligned}
$$


where $\alpha_{k}=2$ if $z_{k} \in B$ and $\alpha_{k}=1$ if $z_{k} \in \partial B, k=1, \ldots, n$. (We indicate a typo in [14]: the indices $k$ and $l$ in the expression $g_{B}\left(z_{k}, z_{l}, \Gamma\right)$ are interchanged.) The quantity in square brackets in the second summand in (1.1) times

$$
\frac{1}{\pi}\left(\sum_{k=1}^{n} \alpha_{k} \delta_{k}^{2}\right)^{-2}
$$

is called the reduced modulus of the domain $B$ relative to $\Gamma$ and $Z, \Delta, \Psi$, and is denoted by $M(B, \Gamma, Z, \Delta, \Psi)$ (see [18]). The following function plays an important part in uniqueness theorems for reduced moduli:

$$
u(z)=\sum_{k=1}^{n} \delta_{k} g_{B}\left(z, z_{k}, \Gamma\right)
$$

it will be called the potential function for $B, \Gamma, Z$, and $\Delta$ (see [19]).

\section{§2. VARiation of the Robin functions under Deformation of a DOMAin OR A PORTION OF ITS BOUNDARY}

First, we consider a general variational theorem for quadratic forms with coefficients that depend on Robin functions and Robin capacities. We restrict ourselves to inner deformations of domains, because the statements for outer deformations differ only by the notation. Let $D$ and $K_{z}$ be finitely connected domains without isolated boundary points. Suppose $D \subset K_{z} \subset \overline{\mathbf{C}}_{z}$, and denote by $K_{w}$ the copy of $K_{z}$ in $\overline{\mathbf{C}}_{w}$. Let $\Gamma$ and $\Gamma^{\prime}$ be closed subsets of $\partial D$ and $\partial K_{z}$ (respectively) consisting of finitely many nondegenerate connected components, let $Z=\left\{z_{k}\right\}_{k=1}^{n}$ be a collection of different admissible points in $\bar{D} \backslash\left(\Gamma \cup \Gamma^{\prime}\right)$, and let $\Delta=\left\{\delta_{k}\right\}_{k=1}^{n}$ be a collection of reals, $\sum_{k=1}^{n} \delta_{k}^{2} \neq 0$. We assume that there exists a function $w=f(z)$ that maps $D$ onto $K_{w}$ conformally and univalently and is such that the derivatives $f^{\prime}\left(z_{k}\right), k=1, \ldots, n$, exist. Let $f(\Gamma)$ be the image of $\Gamma$ in the sense of the boundary mapping principle. By conformal invariance (see 14]), we have

$$
\begin{aligned}
& \sum_{k, l=1}^{n} \alpha_{k} \delta_{k} \delta_{l}\left[g_{D}\left(z_{k}, z_{l}, \Gamma\right)-g_{K_{z}}\left(z_{k}, z_{l}, \Gamma^{\prime}\right)\right] \\
& \quad=\sum_{k, l=1}^{n} \alpha_{k} \delta_{k} \delta_{l}\left[g_{K_{w}}\left(f\left(z_{k}\right), f\left(z_{l}\right), f(\Gamma)\right)-g_{K_{z}}\left(z_{k}, z_{l}, \Gamma^{\prime}\right)\right],
\end{aligned}
$$

where $\alpha_{k}=2$ if $z_{k} \in D$ and $\alpha_{k}=1$ if $z_{k} \in \partial D, k=1, \ldots, n$; for $k=l$ the difference in brackets is defined by the formula

$$
\begin{aligned}
& g_{\varphi(B)}(\varphi(z), \varphi(z), \varphi(\Gamma))-g_{B^{\prime}}\left(z, z, \Gamma^{\prime}\right) \\
& \quad=\lim _{\zeta \rightarrow z}\left[g_{\varphi(B)}(\varphi(\zeta), \varphi(z), \varphi(\Gamma))-g_{B^{\prime}}\left(\zeta, z, \Gamma^{\prime}\right)\right],
\end{aligned}
$$

where $\varphi$ is a map, which may be the identity. Inequalities for the left-hand side of (2.1) can be viewed as monotonicity properties of the quadratic form, whereas inequalities for the right-hand side are qualitative variational principles. Thus, the proof of certain variational principles reduces to potential theory methods.

Theorem 2.1. Suppose $D, K_{z}, K_{w}, \Gamma, \Gamma^{\prime}, Z, \Delta$, and $f$ are as above. Then:

1) if $D=K_{z}$, we have the inequality

$$
\sum_{k, l=1}^{n} \alpha_{k} \delta_{k} \delta_{l}\left[g_{K_{w}}\left(f\left(z_{k}\right), f\left(z_{l}\right), f(\Gamma)\right)-g_{K_{z}}\left(z_{k}, z_{l}, \Gamma^{\prime}\right)\right] \geq 0
$$


in case $\Gamma \subset \Gamma^{\prime}$, and the opposite inequality

$$
\sum_{k, l=1}^{n} \alpha_{k} \delta_{k} \delta_{l}\left[g_{K_{w}}\left(f\left(z_{k}\right), f\left(z_{l}\right), f(\Gamma)\right)-g_{K_{z}}\left(z_{k}, z_{l}, \Gamma^{\prime}\right)\right] \leq 0
$$

in case $\Gamma \supset \Gamma^{\prime}$; moreover, equality occurs if and only if $\Gamma^{\prime}=\Gamma$;

2) if $\overline{(\partial D) \cap K_{z}} \cap\left(\Gamma \cup\left(\bigcup_{k=1}^{n}\left\{z_{k}\right\}\right)\right)=\varnothing$ and $\Gamma=\Gamma^{\prime}$, then again (2.2) is true; moreover, equality occurs if and only if $\bar{D}=\overline{K_{z}}$ and $(\partial D) \cap K_{z}$ consists of finitely many piecewise smooth curves at the interior points of which we have $\partial u / \partial n=0$ for the potential function $u$ constructed by $K_{z}, \Gamma, Z$, and $\Delta$;

3) if $(\partial D) \cap K_{z} \subset \Gamma$ and $\Gamma^{\prime}=\left(\partial K_{z}\right) \backslash((\partial D) \backslash \Gamma)$, then (2.3) is true, and equality occurs if and only if $\bar{D}=\overline{K_{z}}$ and the potential function corresponding to $K_{z}, \Gamma^{\prime}$, $Z$, and $\Delta$ vanishes on $\Gamma \cap K_{z}$.

Proof. The three statements are deduced in a similar way from the monotonicity of the capacity [14, Theorems 1-3], formula (1.1), and the uniqueness theorem of paper [19. Indeed, in case 1), Theorem 1 of [14] yields

$$
\operatorname{cap} C\left(r ; K_{z}, \Gamma^{\prime}, Z, \Delta, \Psi\right) \geq \operatorname{cap} C(r ; D, \Gamma, Z, \Delta, \Psi)
$$

where $\Psi=\{r, \ldots, r\}$, and $r>0$ is sufficiently small. Applying formula (1.1), we obtain

$$
\begin{aligned}
& \sum_{k=1}^{n} \alpha_{k} \delta_{k}^{2} \log r\left(K_{z}, \Gamma^{\prime}, z_{k}\right)+\sum_{k=1}^{n} \sum_{l=1, l \neq k}^{n} \alpha_{k} \delta_{k} \delta_{l} g_{K_{z}}\left(z_{k}, z_{l}, \Gamma^{\prime}\right) \\
& \leq \sum_{k=1}^{n} \alpha_{k} \delta_{k}^{2} \log r\left(D, \Gamma, z_{k}\right)+\sum_{k=1}^{n} \sum_{l=1, l \neq k}^{n} \alpha_{k} \delta_{k} \delta_{l} g_{D}\left(z_{k}, z_{l}, \Gamma\right) ;
\end{aligned}
$$

by $(2.1)$, this is equivalent to $(2.2)$.

Now, if equality occurs in (2.2), then the corresponding reduced moduli are equal:

$$
M\left(K_{z}, \Gamma^{\prime}, Z, \Delta, \Psi\right)=M(D, \Gamma, Z, \Delta, \Psi),
$$

and the sets $\Gamma$ and $\Gamma^{\prime}$ coincide by Theorem 1 in 19 . Cases 2) and 3) are treated similarly, but Theorem 1 in [14] and Theorem 1 in [19] are replaced by other monotonicity results for capacity proved in 14 and other equality cases for reduced moduli discussed in $[19$.

It has already been mentioned that inequalities (2.2) and (2.3) express a sort of extended monotonicity of the Robin capacity. Particular cases of such monotonicity were obtained earlier with the help of the maximum principle for harmonic functions (see 20]), the variational formula for the Green function (see 21]), or the properties of the extremal length for families of curves (see [7, p. 183]). We observe the following. Let either $D, \Gamma, Z$, and $\Delta$ be as earlier, or $D=\overline{\mathbf{C}}_{z}$ and $\Gamma=\varnothing$; suppose that the domain $D \backslash \bigcup_{k=1}^{n}\left\{z_{k}\right\}$ includes a closed subset bounded by curves $\gamma_{j}$ that are determined by the equations $y=g_{j}(x)$, where $g_{1}(x) \leq g_{2}(x), 0 \leq x \leq a, g_{j}(0)=g_{j}(a)=0, j=1,2$. We put $D_{j}=D \backslash \gamma_{j}$. Let $\Gamma_{j}^{+}$be the union of $\Gamma$ and the upper bank of the cut $\gamma_{j}$, and let $\Gamma_{j}^{-}$be the union of $\Gamma$ with the lower bank of the cut $\gamma_{j}$. Repeated application of the monotonicity of the quadratic form in accordance with statements 1) and 2) of Theorem 
2.1 yields the inequalities

$$
\begin{gathered}
\sum_{k, l=1}^{n} \alpha_{k} \delta_{k} \delta_{l}\left[g_{D_{1}}\left(z_{k}, z_{l}, \Gamma_{1}^{+}\right)-g_{D_{2}}\left(z_{k}, z_{l}, \Gamma_{2}^{+}\right)\right] \geq 0 \\
\sum_{k, l=1}^{n} \alpha_{k} \delta_{k} \delta_{l}\left[g_{D_{1}}\left(z_{k}, z_{l}, \Gamma_{1}^{-}\right)-g_{D_{2}}\left(z_{k}, z_{l}, \Gamma_{2}^{-}\right)\right] \leq 0 .
\end{gathered}
$$

Taking Nasyrov's result [11, p. 96] into account, we see that these inequalities are farreaching generalizations of M. A. Lavrent'ev's theorem: if the velocity field has nonnegative gyration, then the body force acting on the arc $\gamma_{2}$ does not exceed that acting on $\gamma_{1}$.

Taking specific canonical domains for the role of $K_{z}$ in Theorem 2.1, we obtain variational principles in the corresponding function classes. We consider some examples, with comments and supplements. Suppose $f \in \mathcal{V}_{1}, D(f) \subset \Delta_{z}$, and $\Gamma$ is a subset of the unit circle $|z|=1$ consisting of finitely many arcs and included in $\partial D(f)$. Statement 2) of Theorem 2.1 leads to a distortion theorem in which the ratio $\left|f^{\prime}\left(z_{k}\right) / f\left(z_{k}\right)\right|$ is estimated in terms of the inner radii and Green functions of the domains $\overline{\mathbf{C}}_{z} \backslash \Gamma$ and $\overline{\mathbf{C}}_{w} \backslash f(\Gamma)$. This case was considered in detail in [22, Theorem 2]. In particular, in that paper it was explained that, under the additional assumptions $n=1, z_{1}=\infty$, and $\delta_{1}=1$, inequality (2.2) (with $\Gamma=\Gamma^{\prime}$ ) coincides with the Pommerenke inequality

$$
\sqrt{\left|f^{\prime}(\infty)\right|} \operatorname{cap} \Gamma \geq \operatorname{cap} f(\Gamma)
$$

see [23, p. 217] (here $\operatorname{cap}(\cdot)$ stands for the logarithmic capacity; the corresponding statement in 23] was stated for functions bounded in the disk and was established under weaker restrictions on $f$ ). If the $\operatorname{arc} \Gamma$ shrinks to a boundary point $z$ at which $f^{\prime}(z)$ exists, the Pommerenke inequality yields

$$
\sqrt{\left|f^{\prime}(\infty)\right|} \geq\left|f^{\prime}(z)\right|
$$

which refines the classical principle mentioned at the beginning of the Introduction and coinciding with the boundary Schwarz lemma. Still stronger refinements can be deduced from Lebedev's paper [24] (see also [25]) Any refinements of and supplements to the Schwarz lemma lead to stronger variational principles; in particular, results of this sort follow from [25, 26]. Here we restrict ourselves to two supplements to (2.4).

Statement 2.1. Suppose $f \in \mathcal{V}_{1}$ and $D(f) \subset \Delta_{z}$. Let $f$ map an arc $\Gamma$ of the circle $|z|=1$ onto the arc $f(\Gamma)$ of the circle $|w|=1$ in the sense of the boundary mapping principle. Then at the point $z$ mapped to the center of the arc $f(\Gamma)$ we have

$$
\left|f^{\prime}(z)\right| \geq\left|f^{\prime}(\infty)\right|^{1 /\left(2 \sin ^{2}(\theta / 4)\right)}
$$

where $\theta$ is the length of the arc $f(\Gamma)$.

In essence, this statement coincides with Theorem 3 in 25]. The next statement follows from inequality (36) in [26] rewritten in terms of the class $\mathcal{V}_{1}$.

Statement 2.2. Suppose $f \in \mathcal{V}_{1}$ and $D(f) \subset \Delta_{z}$. Let the boundary of $D(f)$ include an open arc of the circle $|z|=1$ centered at $z=1$. Suppose $f(1)=1$. Then the Schwarz derivative $S_{f}(z)$ for $f$ obeys the inequality

$$
\operatorname{Re} S_{f}(1) \geq 0 \text {. }
$$

Some estimates of the Schwarz derivative $S_{f}(z)$ can be deduced also from Theorem 2.1 (cf. [18, 22]).

Suppose $f \in \mathcal{V}_{2}$ and $D(f) \subset H_{z}$. Then $f$ can be regarded as the complex potential of an infinitely deep flow over a plane bottom and around the obstacle $H_{z} \backslash D(f)$, with 
hydrodynamical normalization (which means that the flow is unperturbed at infinity). We present the simplest consequences of Theorem 2.1 .

Corollary 2.1. If $f \in \mathcal{V}_{2}, D(f) \subset H_{z}$, and $\Gamma$ is a bounded closed subset of $\partial D(f)$ satisfying $\Gamma \cap \overline{H_{z} \backslash D(f)}=\varnothing$, then

$$
\operatorname{cap} f(\Gamma) \leq \operatorname{cap} \Gamma \text {. }
$$

Proof. The claim follows from Theorem 2.1 (case 2 ) with $n=1, z_{1}=\infty$ : observe that the "Robin function" of the upper half-plane with pole at infinity (relative to $\Gamma$; respectively, $f(\Gamma))$ is the restriction to the upper half-plane of the Green function of the domain $\overline{\mathbf{C}}_{z} \backslash \Gamma$ (respectively, $\overline{\mathbf{C}}_{w} \backslash f(\Gamma)$ ) with pole at $z=\infty$. The inequality written above involves the classical estimate $f^{\prime}(z) \leq 1$ valid for all points $z$ on the boundary of $D(f)$ that lie far apart from the obstacle $H_{z} \backslash D(f)$ (see [2, Subsection 61]).

Corollary 2.2. Suppose $f \in \mathcal{V}_{2}, D(f) \subset H_{z}$, and $\beta_{1}, \beta_{2}$ are real numbers with $\beta_{1} \leq \beta_{2}$. If $\left[\beta_{1}, \beta_{2}\right] \cap \overline{H_{z} \backslash D(f)}=\varnothing$, then for every point $z$ lying on the real axis and not belonging to $\left[\beta_{1}, \beta_{2}\right] \cup\left(\overline{\left.H_{z} \backslash D(f)\right)}\right.$ we have

$$
f^{\prime}(z) \Phi\left(z ; \beta_{1}, \beta_{2}\right) \leq \Phi\left(f(z) ; f\left(\beta_{1}\right), f\left(\beta_{2}\right)\right),
$$

where $\Phi\left(\zeta ; s_{1}, s_{2}\right)=\left(\zeta-s_{1}\right)\left(\zeta-s_{2}\right)\left(\sqrt{\left|\zeta-s_{1}\right|}+\sqrt{\left|\zeta-s_{2}\right|}\right)^{-4}$ and the square roots are assumed to be positive. But if $\left[\beta_{1}, \beta_{2}\right]$ contains all real points of $\overline{H_{z} \backslash D(f)}$, then for every real $z$ apart from $\left[\beta_{1}, \beta_{2}\right]$ we have the opposite inequality.

Proof. Suppose the first assumption of Corollary 2.2 is fulfilled. We put $D=D(f)$, $K_{z}=H_{z}, K_{w}=H_{w}, \Gamma=\Gamma^{\prime}=\left[\beta_{1}, \beta_{2}\right], Z=\{z, \infty\}$, and $\triangle=\{-1,1\}$ in Theorem 2.1. By the second claim of that theorem, inequality (2.2) holds true. In order to calculate the Robin functions on the left in (2.2), we use the fact that they are conformally invariant. Under an appropriate choice of the square root, the function

$$
\zeta=\sqrt{\frac{z-s_{2}}{z-s_{1}}} \quad\left(s_{1}<s_{2}\right)
$$

maps the half-plane $H_{z}$ onto the first quadrant $\Omega$ conformally and univalently in such a way that the segment $\left[s_{1}, s_{2}\right]$ is taken onto the ray $\widetilde{\Gamma}=\{\zeta: \operatorname{Re} \zeta=0, \operatorname{Im} \zeta \geq 0\}$. By symmetry, we have

$$
g_{\Omega}\left(\zeta, \zeta^{\prime}, \widetilde{\Gamma}\right)=\log \left|\frac{\zeta+\zeta^{\prime}}{\zeta-\zeta^{\prime}}\right|
$$

for positive $\zeta^{\prime}$. Putting first $s_{k}=\beta_{k}, k=1,2$, and then $s_{k}=f\left(\beta_{k}\right), k=1,2$, we easily find all parameters of the left-hand side of (2.2). The proof of the second claim of Corollary 2.2 is similar, but the third statement of Theorem 2.1 is employed.

Similar statements can be proved for other classes of functions defined in the Introduction. Some of these results admit equivalent reformulations in various function classes, because of the existence of an appropriate conformal mapping. For instance, for the functions of class $\mathcal{V}_{3}$ (that is, for the complex potentials of flows in curvilinear channels; see [2, Subsections 48 and 62]), we have the following statement.

Corollary 2.3. Suppose $f \in \mathcal{V}_{3}$ and $D(f) \subset S_{z}$. If the segment $\left[\beta_{1}, \beta_{2}\right]$ is included in $(\partial D(f)) \cap\left(\partial S_{z}\right)$, and $\left[\beta_{1}, \beta_{2}\right] \cap \overline{S_{z} \backslash D(f)}=\varnothing$, then for every finite point $z$ on the boundary $\partial S_{z}$ but apart from $\left[\beta_{1}, \beta_{2}\right] \cup\left(\overline{S_{z} \backslash D(f)}\right)$ we have

$$
f^{\prime}(z) e^{\pi(f(z)-z)} \Phi\left(e^{\pi z} ; e^{\pi \beta_{1}}, e^{\pi \beta_{2}}\right) \leq \Phi\left(e^{\pi f(z)} ; e^{\pi f\left(\beta_{1}\right)}, e^{\pi f\left(\beta_{2}\right)}\right) .
$$

But if $\left[\beta_{1}, \beta_{2}\right] \supset \overline{\left(S_{z} \backslash D(f)\right)} \cap \partial S_{z}$, then for every finite point $z \in \partial S_{z} \backslash\left[\beta_{1}, \beta_{2}\right]$ we have the opposite inequality. 
With this result, we finish the analysis of Theorem 2.1, though there are many other examples. But the procedure of finding such applications is clear, so we restrict ourselves to the following remark.

Remark. Theorem 2.1 can be supplemented by incorporating the case where $\Gamma=\varnothing$. Then we should assume that $\sum_{k=1}^{n} \alpha_{k} \delta_{k}=0$, and we should employ Neumann functions instead of Robin functions.

\section{§3. Dilation inequalities And COMPARISON OF DistorTions}

In accordance with classical variational principles, dilation at the stable boundary points reduces under an inner deformation of a domain. It is natural to assume, for instance, that the dilation change becomes smaller as a point recedes away from the deformation. Comparison of dilations at different points for a given deformation can be viewed sometimes as comparison of deformations as they recede away from a given point. Next, comparison of deformations in the extent to which they influence dilation and other characteristics of a given function is interesting for its own sake. We consider some of these questions for the function classes introduced above.

Theorem 3.1. Suppose that either $f \in \mathcal{V}_{2}$ or $f \in \mathcal{V}_{3}$. Let $K_{z}=H_{z}$ in the first case and $K_{z}=S_{z}$ in the second, let $D=D(f)$, and let $\beta$ be a real number. Suppose also that the inner part of the deformation of $K_{z} \backslash D$ lies in the half-plane $\operatorname{Re} z<\beta$, and the outer part $D \backslash K_{z}$ lies in the half-plane $\operatorname{Re} z>\beta$. Let $\Gamma$ be a closed subset of $\partial D$ consisting of finitely many nondegenerate connected components and possessing the following property: $\Gamma \supset\left\{\partial\left[\left(K_{z} \backslash D\right) \cup\left(D \backslash K_{z}\right)\right]\right\} \backslash \partial K_{z}$, and $-\bar{z}+2 \beta \in \Gamma \cup\left(\overline{\mathbf{C}}_{z} \backslash \bar{D}\right)$ whenever $z \in \Gamma \cap \partial K_{z}$ and $\operatorname{Re} z \geq \beta$. Let $Z=\left\{z_{k}\right\}_{k=1}^{n}$ be a collection of distinct admissible points in $\bar{D} \backslash \Gamma$, and let $\Delta=\left\{\delta_{k}\right\}_{k=1}^{n}$ be a collection of positive numbers. Then

$$
\sum_{k, l=1}^{n} \alpha_{k} \delta_{k} \delta_{l}\left[g_{K_{w}}\left(f\left(z_{k}^{\prime}\right), f\left(z_{l}^{\prime}\right), f(\Gamma)\right)-g_{K_{w}}\left(f\left(z_{k}\right), f\left(z_{l}\right), f(\Gamma)\right)\right] \geq 0,
$$

where $\alpha_{k}=2$ if $z_{k} \in D, \alpha_{k}=1$ if $z_{k} \in \partial D$, and the points $z_{k}^{\prime}, k=1, \ldots, n$, are defined as follows: if the point symmetric to $z_{k} \in Z$ with respect to the line $\operatorname{Re} z=\beta$ belongs to $Z$ (and is equal to $z_{l}$ ), then $z_{k}^{\prime}=z_{k}$ if either $\operatorname{Re} z_{k} \geq \beta, \delta_{k} \geq \delta_{l}$ or $\operatorname{Re} z_{k} \leq \beta, \delta_{k} \leq \delta_{l}$, and $z_{k}^{\prime}=z_{l}$ otherwise (i.e., either $\operatorname{Re} z_{k} \geq \beta, \delta_{k}<\delta_{l}$ or $\operatorname{Re} z_{k} \leq \beta, \delta_{k}>\delta_{l}$ ). But if the symmetric point $-\overline{z_{k}}+2 \beta$ does not belong to $Z$, then $z_{k}^{\prime}=z_{k}$ if $\operatorname{Re} z_{k} \geq \beta$ and $z_{k}^{\prime}=-\overline{z_{k}}+2 \beta$ if $\operatorname{Re} z_{k} \leq \beta$.

Proof. We use the polarization of condensers techniques (see [5]). Let $\Psi=\{r, \ldots, r\}$, where $r>0$ is sufficiently small, and let $u$ be an admissible function for the condenser $C(r ; D, \Gamma, Z, \Delta, \Psi)$, extended by 0 to $\overline{\mathbf{C}}_{z} \backslash \bar{D}$. Then the function

$$
u^{\prime}(z)= \begin{cases}\max [u(z), u(-\bar{z}+2 \beta)] & \text { if } \operatorname{Re} z \geq \beta \\ \min [u(z), u(-\bar{z}+2 \beta)] & \text { if } \operatorname{Re} z \leq \beta\end{cases}
$$

is admissible for the condenser $C\left(r ; D, \Gamma, Z^{\prime}, \Delta, \Psi\right)$, where $Z^{\prime}=\left\{z_{k}^{\prime}\right\}_{k=1}^{n}$, and moreover, $I(u, D)=I\left(u^{\prime}, D\right)$ (cf. [5, p. 16]). It follows that

$$
\operatorname{cap} C(r ; D, \Gamma, Z, \Delta, \Psi)=I(u, D) \geq \operatorname{cap} C\left(r ; D, \Gamma, Z^{\prime}, \Delta, \Psi\right) .
$$

Applying formula (1.1), we obtain

$$
\sum_{k, l=1}^{n} \alpha_{k} \delta_{k} \delta_{l}\left[g_{D}\left(z_{k}^{\prime}, z_{l}^{\prime}, \Gamma\right)-g_{D}\left(z_{k}, z_{l}, \Gamma\right)\right] \geq 0
$$

which is equivalent to (3.1) by conformal invariance. 
By the procedures of the paper [19, it can be shown that, for a nondegenerate deformation, equality in (3.1) occurs only in the case where $z_{k}=z_{k}^{\prime}, k=1, \ldots, n$. For reduced moduli, inequalities of the form (3.1) were studied earlier in [5]. Similar inequalities were proved by Solynin [27] for Green functions, and by Betsakos [28] for Robin functions. The methods of 27] and [28] differ from the proof of Theorem 3.1. We illustrate inequality (3.1) by the following simple examples.

Corollary 3.1. Suppose $f \in \mathcal{V}_{2}, D(f) \subset H_{z}$, and $H_{z} \backslash D(f) \subset\{z: \operatorname{Re} z<\sigma\}$. Then for any two real points $z_{1}, z_{2}$ with $\sigma<z_{1}<z_{2}$ we have

$$
\frac{f^{\prime}\left(z_{1}\right)}{f\left(z_{1}\right)-f(\sigma)} \geq \frac{f^{\prime}\left(z_{2}\right)}{f\left(z_{2}\right)-f(\sigma)} .
$$

Proof. In Theorem 3.1, put $\beta=\left(z_{1}+z_{2}\right) / 2$ and let $\Gamma$ coincide with the part of the boundary $\partial D(f)$ from $z=\infty$ to $z=\sigma$, oriented in accordance with the positive orientation of the boundary. Let $Z=\left\{z_{1}\right\}, \Delta=\{1\}(n=1)$. Then $z_{2}=z_{1}^{\prime}$, and inequality (3.1) yields

$$
g_{H_{w}}\left(f\left(z_{2}\right), f\left(z_{2}\right),[-\infty, f(\sigma)]\right)-g_{H_{w}}\left(f\left(z_{1}\right), f\left(z_{1}\right),[-\infty, f(\sigma)]\right) \geq 0,
$$

where $[-\infty, f(\sigma)]$ is a ray on the real axis. A direct calculation of Robin capacities leads to the required inequality.

Corollary 3.2. Suppose $f \in \mathcal{V}_{2}, D(f) \subset H_{z}$, and $H_{z} \backslash D(f) \subset\{z: \operatorname{Re} z<0\}$. Then for every positive $z_{1}, z_{2}$, and $y$ with $z_{1}<z_{2}$ we have

$$
\begin{aligned}
\operatorname{Im} f\left(z_{1}+i y\right) & \leq \operatorname{Im} f\left(z_{2}+i y\right), \\
f^{\prime}\left(z_{1}\right) & \leq f^{\prime}\left(z_{2}\right) .
\end{aligned}
$$

Proof. In Theorem 3.1, put $\beta=\left(z_{1}+z_{2}\right) / 2, \Gamma=\partial D(f), Z=\left\{z_{1}+i y, z^{*}\right\}, z^{*}=$ $\left(z_{1}+z_{2}\right) / 2+i t$, and $\Delta=\{\delta, 1\}(t>0, \delta>0)$. Inequality (3.1) implies that

$$
\begin{aligned}
& \delta g_{H_{w}}\left(f\left(z_{1}+i y\right), f\left(z^{*}\right), f(\Gamma)\right)+\delta^{2} \log r\left(D(f), \Gamma, z_{1}+i y\right) \\
& \quad \leq \delta g_{H_{w}}\left(f\left(z_{2}+i y\right), f\left(z^{*}\right), f(\Gamma)\right)+\delta^{2} \log r\left(D(f), \Gamma, z_{2}+i y\right) .
\end{aligned}
$$

Dividing by $\delta$ and passing to the limit as $\delta \rightarrow 0$, we obtain

$$
g_{H_{w}}\left(f\left(z_{1}+i y\right), f\left(z^{*}\right), f(\Gamma)\right) \leq g_{H_{w}}\left(f\left(z_{2}+i y\right), f\left(z^{*}\right), f(\Gamma)\right)
$$

or, which is the same,

$$
\left|\frac{f\left(z_{1}+i y\right)-\overline{f\left(z^{*}\right)}}{f\left(z_{1}+i y\right)-f\left(z^{*}\right)}\right| \leq\left|\frac{f\left(z_{2}+i y\right)-\overline{f\left(z^{*}\right)}}{f\left(z_{2}+i y\right)-f\left(z^{*}\right)}\right| .
$$

Taking the asymptotic expansion of $f$ near infinity into account, we deduce the first inequality. The second inequality follows from the first.

Thus, under the assumptions of Corollary 3.2 (and in terms of the complex potential), regardless of the form of the obstacle $H_{z} \backslash D(f)$, to the right of the imaginary axis the streamlines are lowering monotonically, and the flow velocity is monotone nondecreasing along the real axis. Invoking the Hopf lemma, we can check, along the lines of the proof of Corollary 3.2, that the velocity is strictly monotone increasing on the real axis. Similar statements for derivatives at boundary points were used efficiently by Solynin and other authors for the solution of "minimal area" problems in various settings (see, e.g., [29, 30]).

Theorem 3.2. Suppose $f \in \mathcal{V}_{5}, D(f) \subset K_{z}(R)$, and some boundary component of $D(f)$ coincides with the circle $|z|=1$. Let $Z=\left\{z_{k}\right\}_{k=1}^{n}$ be a collection of different admissible points in $D(f) \cup\{z:|z|=1\}$, and let $\Delta=\left\{\delta_{k}\right\}_{k=1}^{n}$ be a collection of nonzero reals 
satisfying the following condition: on every circle $|z|=\rho, 1 \leq \rho<R$, there are at most two points of $Z$, and moreover, if $\left|z_{k}\right|=\left|z_{l}\right|$, then $\delta_{k} \delta_{l}<0$. Then

$$
\sum_{k, l=1}^{n} \alpha_{k} \delta_{k} \delta_{l}\left[g_{K_{z}(R)}\left(z_{k}^{*}, z_{l}^{*}, \Gamma_{2}\right)-g_{K_{w}(R(f))}\left(f\left(z_{k}\right), f\left(z_{l}\right), \Gamma_{1}\right)\right] \geq 0,
$$

where the $\alpha_{k}$ are as before, $z_{k}^{*}=\left|z_{k}\right|$ if $\delta_{k}>0$ and $z_{k}^{*}=-\left|z_{k}\right|$ if $\delta_{k}<0, k=1, \ldots, n$; $\Gamma_{2}=\{z:|z|=R\}, \Gamma_{1}=\{w:|w|=R(f)\}$.

Proof. Let $\Gamma$ be the "outer" boundary component of the domain $D(f)$, let $\Psi=\{r, \ldots, r\}$, and let $u$ be the potential function of the condenser $C(r ; D, \Gamma, Z, \Delta, \Psi)$, extended by 0 to the annulus $\overline{K_{z}}(R)$. We denote by $u^{*}$ the result of circular symmetrization of $u$ with respect to the real semiaxis; see [5]. It is easily seen that $u^{*}$ is an admissible function for the condenser $C\left(r ; K_{z}(R), \Gamma_{2}, Z^{*}, \Delta, \Psi\right)$, where $Z^{*}=\left\{z_{k}^{*}\right\}_{k=1}^{n}$ and $r>0$ is sufficiently small. Taking the Pólya-Szegö inequality into account, we obtain

$$
\begin{aligned}
& \operatorname{cap} C(r ; D, \Gamma, Z, \Delta, \Psi) \\
& \quad=I(u, D) \geq I\left(u^{*}, K_{z}(R)\right) \geq \operatorname{cap} C\left(r ; K_{z}(R), \Gamma_{2}, Z^{*}, \Delta, \Psi\right) .
\end{aligned}
$$

It remains to refer to formula (1.1).

This approach involving circular or Steiner symmetrization makes it possible to obtain similar results for functions in the classes $\mathcal{V}_{1}-\mathcal{V}_{3}$. Now we present an example of a different nature. If $f \in \mathcal{V}_{4}(l)$, then $D(f)$ can be viewed, e.g., as a filtration domain, $f / L(f)$ can be viewed as the complex potential of the filtration flow, and $1 / L(f)$ can be viewed as the consumption of liquid. Consider the condenser $C(f)=\left(D(f),\left\{a_{4} a_{1}, a_{2} a_{3}\right\},\{0,1\}\right)$. Since a condenser's capacity is monotone, we see that if the edge $a_{1} a_{2}$ coincides with the segment $[l, l+i]$ and the edge $a_{3} a_{4}$ coincides with the segment $[i, 0]$, then $L(f) \geq l$ provided $D(f) \subset Q_{z}(l, l+i, i, 0)$, and $L(f) \leq l$ provided $D(f) \supset Q_{z}(l, l+i, i, 0)$. In the next theorem it is shown that inner deformations of a quadrangle have greater influence on $L(f)$ than outer deformations of similar type.

Theorem 3.3. If $f \in \mathcal{V}_{4}(l), D(f) \subset \Pi:=\{z: 0 \leq \operatorname{Re} z \leq l\}$, the segments $[l, l+i]$ and $[i, 0]$ are edges of the quadrangle $D(f)$, and the other two edges are obtained from each other by a shift along the imaginary axis, then $l \leq L(f)$.

Now, suppose $f_{k} \in \mathcal{V}_{4}(l), D\left(f_{k}\right) \subset \Pi, k=1,2$, and the corresponding edges of the quadrangles $D\left(f_{1}\right)$ and $D\left(f_{2}\right)$ are the segments $[l, l+i]$ and $[i, 0]$. Next, suppose that the other two edges of $D\left(f_{1}\right)$ are some curves $\gamma_{1}$ and $\gamma_{2}\left(0 \in \gamma_{1}, l \in \gamma_{1}\right)$ and that the edges of the quadrangle $D\left(f_{2}\right)$ are obtained from those of $D\left(f_{1}\right)$ by reflection of $\gamma_{1}$ with respect to the real axis, and of $\gamma_{2}$ with respect to the line $\operatorname{Im} z=1$. Then $l \leq\left(L\left(f_{1}\right)+L\left(f_{2}\right)\right) / 2$.

Finally, if $f \in \mathcal{V}_{4}(l), D(f) \subset \Pi$, the segments $[l, l+i]$ and $[i, 0]$ are edges of the quadrangle $D(f)$ and each of the other two edges is centrally symmetric $\left(a_{4} a_{1}\right.$ with respect to the point $l / 2$ and $a_{2} a_{3}$ with respect to the point $\left.l / 2+i\right)$, then $l \leq L(f)$.

Proof. The first claim follows from the properties of "symmetrization of a quadrangle" (see [31]). The second claim can easily be deduced from Marcus' linear averaging transformation (see [32]). Indeed, let $u_{1}$ be the potential function for the condenser $C\left(f_{1}\right)$, and let $u_{2}$ be that for $C\left(f_{2}\right)$. We extend these functions by 0 and 1 so as to make them continuous in the entire strip $\Pi$. Denote by $u^{*}$ the result of the linear averaging transformation applied either to the functions $u_{1}, u_{2}$, or to their shifts along the imaginary axis chosen in such a way that they fit formally in the framework of the definition in 32 (the numbers $1 / 2,1 / 2$ are chosen as weights). The function $u^{*}$ may differ only by a shift from the potential function of the rectilinear quadrangle $Q_{z}(l, l+i, i, 0)$. By the Marcus 
theorem, we have

$$
I\left(u_{1}, D\left(f_{1}\right)\right)+I\left(u_{2}, D\left(f_{2}\right)\right) \geq 2 I\left(u^{*}, Q_{z}(l, l+i, i, 0)\right) \geq 2 l,
$$

which completes the proof of the second claim because cap $C\left(f_{k}\right)=L\left(f_{k}\right), k=1,2$. To prove the third claim, we consider the functions $u_{1}(z)=u(z)$ and $u_{2}(z)=u(-\bar{z}+l)$ extended by 0 and by 1 to the entire strip $\Pi^{\prime}=\{z: 0 \leq \operatorname{Re} z \leq l / 2\}$. Here $u(z)$ is the potential function for the condenser $C(f)$. We again apply the linear averaging transformation (with weights $(1 / 2,1 / 2))$ that takes the pair $\left\{u_{1}, u_{2}\right\}$ to $u^{*}$; this yields

$$
\operatorname{cap} C(f)=I(u, D(f))=I\left(u_{1}, \Pi^{\prime}\right)+I\left(u_{2}, \Pi^{\prime}\right) \geq 2 I\left(u^{*}, \Pi^{\prime}\right) \geq l .
$$

Similar statements for functions in the classes $\mathcal{V}_{1}$ and $\mathcal{V}_{5}$ are deduced by using radial averaging transformations (see [32]). In addition to Theorem 3.3, we note that in the recent paper 33 . some theorems of variational nature were obtained for rectilinear quadrangles. Some of these statements remain valid if one edge is replaced with a curve. For example, Theorem 5.3 in 33 on the variation of the conformal modulus of a quadrangle under rotation of one edge remains valid if the "upper edge" is replaced with the curve $y=y(x)$, where $y(x), 0 \leq x \leq 1$, is a monotone increasing function. These examples do not even approach the entire scope of applications of polarization, symmetrization, and dissymmetrization (see [5]) to variational principles of conformal mapping. Here we only discuss approaches to such problems in the example of the function classes chosen.

\section{§4. BehaVior OF LEVEL CURVES}

For a function $f$ of class $\mathcal{V}_{1}$ or $\mathcal{V}_{5}$, the term level curve usually means a closed analytic curve $C_{\rho}(f)$ defined by the equation $|f(z)|=\rho$; here $\rho>1$ for $\mathcal{V}_{1}$ and $1<\rho<R$ for $\mathcal{V}_{2}$. If $f$ belongs either to $\mathcal{V}_{2}$, or to $\mathcal{V}_{3}$, or to $\mathcal{V}_{4}(l)$, then a level curve $C_{v}(f)$ is defined by the equation $\operatorname{Im} f(z)=v$, where $v>0$ for $\mathcal{V}_{2}$ and $0<v<1$ in the remaining cases. Here we present certain statements for inner deformations. These statements are obtained by various methods of geometric function theory and refine widely known variational principles of conformal mappings (cf. 2, Subsection 61, the first claims in Theorems 1-3]). All these statements can also be proved by using symmetrization (see [5]), which will be shown by an example of two proofs. We denote by $z=p(w ; \alpha), 0<\alpha \leq 1$, the Pick function defined by the equation

$$
\frac{z}{(1-z)^{2}}=\frac{\alpha w}{(1-w)^{2}}, \quad w \in \Delta_{w}
$$

taking the domain $\Delta_{w}$ onto the domain $\Delta_{z}$ cut along the segment $[p(-1 ; \alpha),-1]$. Next, for any two numbers $R_{1}$ and $R_{2}$ with $1<R_{1} \leq R_{2}<\infty$, we denote by $z=r\left(w ; R_{1}, R_{2}\right)$ the function that maps the annulus $K_{w}\left(R_{1}\right)$ conformally and univalently onto the annulus $K_{z}\left(R_{2}\right)$ cut along the segment $\left[-R_{2}, r\left(-R_{1} ; R_{1}, R_{2}\right)\right]$ on the real line.

Statement 4.1. If $f \in \mathcal{V}_{1}, f^{\prime}(\infty)=\alpha>0$, and $D(f) \subset \Delta_{z}$, then for every $\rho>1$ the level curves $C_{\rho}(f)$ lie in the annulus

$$
p(\rho ; \alpha) \leq|z| \leq-p(-\rho ; \alpha) .
$$

If for some $\rho>1$ the curve $C_{\rho}(f)$ touches the boundary of the annulus, then for some real $\varphi$ we have $p\left(e^{-i \varphi} f\left(z e^{i \varphi}\right) ; \alpha\right) \equiv z$.

Statement 4.2. Suppose $f \in \mathcal{V}_{5}, D(f) \subset K_{z}(R)$, and $R(f) \leq R$. Then for every $\rho$ with $1<\rho<R(f)$, the level curve $C_{\rho}(f)$ lies in the set

$$
-r(-\rho ; R(f), R) \leq|z| .
$$


If, moreover, some boundary component of $D(f)$ is the circle $|z|=1$, then the level curve $C_{\rho}(f)$ also lies in the disk

$$
|z| \leq r(\rho ; R(f), R)
$$

Under the above conditions, if for some $\rho, 1<\rho<R(f)$, the curve $C_{\rho}(f)$ touches the boundary of the sets (4.1) or (4.2) (respectively), then for some real $\varphi_{1}$ and $\varphi_{2}$ we have $r\left(e^{i \varphi_{2}} f\left(z e^{i \varphi_{1}}\right) ; R(f), R\right) \equiv z$.

Statement 4.1 is equivalent to the Pick theorem for bounded univalent functions (see, e.g., [34, p. 55]). Passing to Statement 4.2, we observe first that Kubo's result (see 35, p. 65]) and standard arguments imply that $C_{\rho}(f)$ is included in the set (4.1). Kubo's proof leans upon circular symmetrization, which, moreover, makes it possible to find out whether a level curve $C_{\rho}(f)$ touches the boundary of the set (4.1) (see [36, 37]). The fact that $C_{\rho}(f)$ is included in the disk (4.2) can by proved by circular symmetrization of quadrangles (see [36]). Here we present a detailed proof in terms of the condensers' capacity. Fix a point $z_{0}$ on $C_{\rho}(f)$. On the plane $\mathbf{C}_{w}$, we consider the condenser $C=\left(K_{w}(R(f)) \backslash(-R(f),-1),\{\{w:|w|=R(f)\} \cup(-R(f),-1],[1, \rho]\},\{0,1\}\right)$. By conformal invariance, the capacity of $C$ is equal to that of the condensers $C_{1}=\left(K_{z}(R),\{\{z\right.$ : $|z|=R\} \cup(-R,-1],[1, r(\rho ; R(f), R)]\},\{0,1\})$ and $C_{2}=\left(D(f) \backslash f^{-1}(-R(f),-1)\right.$, $\left.\left\{[(\partial D(f)) \backslash\{z:|z|=1\}] \cup f^{-1}((-R(f),-1]), f^{-1}([1, \rho])\right\},\{0,1\}\right)$. Let $u(z)$ be the potential function of the condenser $C_{2}$, and let $u^{*}(z)$ be the result of the circular symmetrization of $u$ relative to the positive semiaxis (see [5]). It is easily seen that $u^{*}$ is admissible for the condenser $C_{3}=\left(K_{z}(R),\left\{\{z:|z|=R\} \cup[-R,-1],\left[1,\left|z_{0}\right|\right]\right\},\{0,1\}\right)$. Combined with Theorem 1.3 in [5], this yields consecutively

$$
\operatorname{cap} C_{1}=\operatorname{cap} C=\operatorname{cap} C_{2}=I(u, D(f)) \geq I\left(u^{*}, K_{z}(R)\right) \geq \operatorname{cap} C_{3} .
$$

Since the capacity is monotone (see [14]), it follows that

$$
\left|z_{0}\right| \leq r(\rho ; R(f), R)
$$

moreover, in the equality case (i.e., when $C_{\rho}(f)$ touches the boundary of the disk (4.2)), $C_{1}$ coincides with $C_{2}$ up to a rotation about the origin (see [37, pp. 177-179]). This implies the identity $r\left(e^{i \varphi_{1}} f\left(z e^{i \varphi_{2}}\right) ; R(f), R\right) \equiv z$ for some real $\varphi_{1}$ and $\varphi_{2}$, as required.

Now we introduce the following notation. For $a \geq 0$, let

$$
z=s(w ; a) \equiv \frac{2}{\pi} \cosh ^{-1}\left(e^{-a \pi / 4} \cosh (\pi w / 2)\right)
$$

be the function that maps the strip $S_{w}$ conformally and univalently onto the strip $S_{z}$ cut along the segment $[0, s(0, a)]$ on the imaginary axis. For fixed $l_{1}$ and $l_{2}, l_{1} \leq l_{2}$, let $z=q\left(w ; l_{1}, l_{2}\right)$ denote the function that maps the rectilinear quadrangle $Q_{w}\left(l_{2}, l_{2}+i, i, 0\right)$ conformally and univalently onto the rectangle $0<\operatorname{Re} z<l_{1}, 0<\operatorname{Im} z<1$ in such a way that the point $q\left(l_{2} ; l_{1}, l_{2}\right)$ lies on the segment $\left[l_{1}, l_{1}+i\right], q\left(l_{2}+i ; l_{1}, l_{2}\right)=l_{1}+i$, $q\left(i ; l_{1}, l_{2}\right)=i$, and $q\left(0 ; l_{1}, l_{2}\right)=0$. This function is determined by the relations

$$
\begin{gathered}
\omega=\operatorname{sn}\left[w K\left(\sqrt{1-\tau^{2}}\right)-K(\tau) ; \tau\right], \quad \zeta=\operatorname{sn}\left[z K\left(\sqrt{1-k^{2}}\right)-K(k) ; k\right], \\
\frac{\omega \tau+1}{\omega \tau-1} \cdot \frac{\tau+1}{\tau-1}=\frac{\zeta k+1}{\zeta k-1} \cdot \frac{k+1}{k-1},
\end{gathered}
$$

where $k$ and $\tau$ are solutions of the equations

$$
l_{1}=2 K(k) / K\left(\sqrt{1-k^{2}}\right)
$$

and

$$
l_{2}=2 K(\tau) / K\left(\sqrt{1-\tau^{2}}\right)
$$


respectively, $K(t)$ is the full elliptic integral of the first kind with modulus $t$, and $\operatorname{sn}[\cdot ; t]$ is the elliptic sine. We observe that the function $r\left(w ; R_{1}, R_{2}\right)$ introduced earlier is representable in terms of $q\left(w ; l_{1}, l_{2}\right)$ in the following way:

$$
\begin{aligned}
& r\left(w ; R_{1}, R_{2}\right)=\exp \left\{\left(\log R_{2}\right)\left[i q\left(-i\left(\frac{\log w}{\log R_{1}}-1\right) ; \frac{\pi}{\log R_{2}}, \frac{\pi}{\log R_{1}}\right)+1\right]\right\}, \\
& r\left(1 ; R_{1}, R_{2}\right)=1 .
\end{aligned}
$$

Statement 4.3. If $f \in \mathcal{V}_{2}, f(z)=z+c / z+o(1 / z)$ as $z \rightarrow \infty(z \in D(f))$, and $D(f) \subset H_{z}$, then for every $v>0$ the level curve $C_{v}(f)$ lies in the strip

$$
v \leq \operatorname{Im} z \leq \sqrt{v^{2}+2 c} .
$$

If for some $v>0$ the curve $C_{v}(f)$ touches the lower boundary of the strip, then $f(z) \equiv z$ $(c=0)$; if it touches the upper boundary, then $f(z)=\sqrt{z^{2}+2 c}$, and the contact point is $i \sqrt{2 c}, c \geq 0$.

Statement 4.4. Suppose $f \in \mathcal{V}_{3}, D(f) \subset S_{z}$, and

$$
\lim _{\substack{z \rightarrow+\infty \\ z \in D}}(f(z)-z)-\lim _{\substack{z \rightarrow-\infty \\ z \in D}}(f(z)-z)=a .
$$

Then for every $v, 0<v<1$, the level curve $C_{v}(f)$ lies in the half-plane

$$
\operatorname{Im} z \leq \operatorname{Im} s(i v ; a) \text {. }
$$

If, moreover, $\overline{S_{z} \backslash D(f)} \cap\{z: \operatorname{Im} z=1\}=\varnothing$, then the level curve $C_{v}(f)$ also lies in the half-plane

$$
v \leq \operatorname{Im} z .
$$

If for some $v, 0<v<1$, the curve $C_{v}(f)$ touches the boundary of the half-plane (4.4), then $f(z) \equiv z+b$ for some real $b$.

Statement 4.5. If $f \in \mathcal{V}_{4}(l)$ and $D(f) \subset Q_{z}(l, l+i, i, 0)$, then for every $v$ with $0<v<1$ the level curve $C_{v}(f)$ lies in the half-plane

$$
\operatorname{Im} z \leq \operatorname{Im} q(L(f)+i v ; l, L(f)) .
$$

If, moreover, the edge $a_{2} a_{3}$ of the quadrangle $D(f)$ coincides with the segment $[l+i, i]$, then $C_{v}(f)$ also lies in the half-plane

$$
\operatorname{Im} q(i v ; l, L(f)) \leq \operatorname{Im} z .
$$

If for some $v, 0<v<1$, the level curve $C_{v}(f)$ touches the boundary of some of these half-planes, then $C_{v}(f)$ coincides with the curve $z=q(u+i v ; l, L(f)), 0<u<1$, or with its reflection in the line $\operatorname{Re} z=l / 2$.

First, we observe that Statement 4.5 for $\mathcal{V}_{4}(l)$-functions reduces to Statement 4.1 for $\mathcal{V}_{5}$-functions by standard tools involving conformal mapping and the symmetry principle. Next, Statement 4.3 for functions in the class $\mathcal{V}_{2}$ can be established with the help of parametric extension of univalent functions in a half-plane (see 38]). The lower estimate can also be deduced from the maximal principle for harmonic functions (see [2]). It is well known that the level curves lie in the half-plane (4.4); see 2]. Finally, the upper estimate (4.3) is implicit in Goryaĭnov's paper 39] (see Theorem 11 and the proof of Theorem 10 in 39]). We give another proof of this estimate by the symmetrization method. Namely, we show that (4.3) is fulfilled for every point $z_{0}$ of the curve $C_{v}(f)$. There is no loss of generality in assuming that $\operatorname{Re} z_{0}=\operatorname{Re} f\left(z_{0}\right)=0$. We introduce the following notation: $\psi$ is the conformal and univalent map of the strip $S_{z}$ onto the disk $|\zeta|<1$ normalized by the conditions $\psi(-\infty)=-1, \psi(+\infty)=1, \psi(i)=i$. The same letter will be reserved 
for the map of the strip $S_{w}$ onto the disk $|w|<1$ with the same normalization. We put $\zeta_{0}=\psi\left(z_{0}\right), \quad \zeta_{0}^{*}=\psi(s(i v ; a))$ and denote by $\zeta^{\prime}$ the boundary point of $\psi(D(f))$ that lies on the segment $\left[\zeta_{0}, i\right]$ and is the nearest to $\zeta_{0}$. Next, $F=\psi \circ f \circ \psi^{-1}$ is a conformal map of $\psi(D(f))$ onto the disk $|w|<1$. For $r$ sufficiently small, consider the condensers

$$
\begin{aligned}
& C_{1}(r)=C\left(r ;\{\zeta:|\zeta|<1\},\left[\zeta_{0}, i\right],\{-1,1\},\{-1,-1\},\{r, r\}\right), \\
& C_{2}(r)=C\left(r ; \psi(D(f)),\left[\zeta_{0}, \zeta^{\prime}\right],\{-1,1\},\{-1,-1\},\{r, r\}\right), \\
& C_{3}(r)=C\left(r ;\{w:|w|<1\}, F\left(\left[\zeta_{0}, \zeta^{\prime}\right]\right),\{-1,1\},\{-1,-1\},\left\{r\left|F^{\prime}(-1)\right|, r\left|F^{\prime}(1)\right|\right\}\right), \\
& C_{3}^{*}(r)=C\left(r ;\{w:|w|<1\},[\psi(i v), i],\{-1,1\},\{-1,-1\},\left\{r e^{-\pi a / 2}, r e^{-\pi a / 2}\right\}\right), \\
& C_{4}(r)=C\left(r ;\{\zeta:|\zeta|<1\},\left[\zeta_{0}^{*}, i\right],\{-1,1\},\{-1,-1\},\{r, r\}\right) .
\end{aligned}
$$

Since the capacity is monotone (see [14]) and is invariant under $F$, we see that

$$
\operatorname{cap} C_{1}(r) \geq \operatorname{cap} C_{2}(r)=\operatorname{cap} C_{3}(r) \text {. }
$$

Let $u(w)$ be the potential function of the condenser $C_{3}(r)$, let $u^{*}(w)=\operatorname{St} u(\psi(w))$, where St is Steiner's symmetrization with respect to the imaginary axis, and let $u^{\prime}(w)=$ $u^{*}\left(\psi^{-1}(w)\right)$. Then $u^{\prime}(w)$ is an admissible function for the condenser $C_{3}^{*}(r)$, and we have

$$
\begin{aligned}
& \operatorname{cap} C_{3}(r)=I(u,\{w:|w|<1\}) \\
& \quad \geq I\left(u^{*}, S_{w}\right)=I\left(u^{\prime},\{w:|w|<1\}\right) \geq \operatorname{cap} C_{3}^{*}(r)=\operatorname{cap} C_{4}(r) .
\end{aligned}
$$

The last identity follows from the invariance of a condenser's capacity under the map $\psi \circ s(\cdot ; a) \circ \psi^{-1}$. The resulting inequality cap $C_{1}(r) \geq \operatorname{cap} C_{4}(r)$, the asymptotics of the capacity as $r \rightarrow 0$, and the property of the reduced modulus to be monotonic (see [19]) yield $\operatorname{Im} \zeta_{0} \leq \operatorname{Im} \zeta_{0}^{*}$, or, equivalently, $\operatorname{Im} z_{0} \leq \operatorname{Im} s(i v ; a)$. The proof is finished.

The results of 19 make it possible to study the cases where a level curve $C_{v}(f)$ touches the boundary of the half-plane (4.3). It should also be noted that curvature estimates for level curves in the case of $\mathcal{V}_{1}$ or $\mathcal{V}_{2}$ are known; see [1, 38. The paper [40] contains a systematic treatment of functions that map a strip into itself.

\section{REFERENCES}

[1] G. M. Goluzin, Geometric theory of functions of a complex variable, 2nd ed., "Nauka", Moscow, 1966; English transl. of 2nd ed., Transl. Math. Monogr., vol. 26, Amer. Math. Soc., Providence, RI, 1969. MR0219714 (36:2793) MR0247039 (40:308)

[2] M. A. Lavrent'ev and B. V. Shabat, Methods of function theory of a complex variable, "Nauka", Moscow, 1973. (Russian) MR1087298 (91k:30003)

[3] G. V. Kuz'mina, Methods of geometric function theory. I, II, Algebra i Analiz 9 (1997), no. 3, 41103; no. 5, 1-50; English transl., St. Petersburg Math. J. 9 (1998), no. 3, 455-507; no. 5, 889-930. MR 1466796 (98h:30041) MR1604397 (99c:30047a)

[4] S. Bergman and M. Schiffer, Kernel functions and elliptic differential equations in mathematical physics, Academic Press, New York, 1953. MR0054140(14:876d)

[5] V. N. Dubinin, Symmetrization in the geometric theory of functions of a complex variable, Uspekhi Mat. Nauk 49 (1994), no. 1, 3-76; English transl., Russian Math. Surveys 49 (1994), no. 1, 1-79. MR 1307130(96b:30054)

[6] I. I. Lyashko, I. M. Velikoivanenko, V. I. Lavrik, and G. E. Mistetskiı̌, The method of majorant domains in the theory of filtration, "Naukova Dumka", Kiev, 1974. (Russian) MR0464873(57:4794)

[7] P. Duren, Robin capacity, Computational Methods and Function Theory (Nicosia, 97) (N. Papamichael, St. Ruscheweyh, and E. B. Saff, eds.), Ser. Approx. Decompos., vol. 11, World Sci. Publishing, River Edge, NJ, 1999, pp. 177-190. MR.1700346 (2001b:30040)

[8] P. Duren, J. Pfaltzgraff, and R. Thurman, Physical interpretation and further properties of Robin capacity, Algebra i Analiz 9 (1997), no. 3, 211-219; English transl., St. Petersburg Math. J. 9 (1998), no. 3, 607-614. MR1466802 (98k:31002) 
[9] B. Dittmar and A. Yu. Solynin, Distortion of the hyperbolic Robin capacity under a conformal mapping, and extremal configurations, Zap. Nauchn. Sem. S.-Peterburg. Otdel. Mat. Inst. Steklov. (POMI) 263 (2000), 49-69; English transl., J. Math. Sci. (New York) 110 (2002), no. 6, 3058-3069. MR:1756337 (2001f:30030)

[10] M. D. O'Neill and R. E. Thurman, Extremal domains for Robin capacity, Complex Variables Theory Appl. 41 (2000), 91-109. MR1756782 (2001a:30028)

[11] S. Nasyrov, Robin capacity and lift of infinitely thin airfoils, Complex Variables Theory Appl. 47 (2002), no. 2, 93-107. MR1892511 (2003c:30019)

[12] M. Stiemer, A representation formula for the Robin function, Complex Variables Theory Appl. 48 (2003), no. 5, 417-427. MR1974379 (2003m:30056)

[13] A. Yu. Vasil'ev, Robin's modulus in a Hele-Shaw problem, Complex Variables Theory Appl. 49 (2004), no. 7-9, 663-672. MR2088056 (2006a:30025)

[14] V. N. Dubinin, Generalized condensers and the asymptotics of their capacities under a degeneration of some plates, Zap. Nauchn. Sem. S.-Peterburg. Otdel. Mat. Inst. Steklov. (POMI) 302 (2003), 38-51; English transl., J. Math. Sci. (New York) 129 (2005), no. 3, 3835-3842. MR2023031 (2004m:31001)

[15] D. Gaier and W. Hayman, On the computation of modules of long quadrilaterals, Constr. Approx. 7 (1991), 453-467. MR1124970 (93a:30006)

[16] A. Yu. Solynin, Moduli and extremal metric problems, Algebra i Analiz 11 (1999), no. 1, 3-86; English transl., St. Petersburg Math. J. 11 (2000), no. 1, 1-65. MR.1691080(2001b:30058)

[17] V. M. Miklyukov, Conformal mapping of a nonregular surface and its applications, Volgograd. Gos. Univ., Volgograd, 2005. (Russian)

[18] V. N. Dubinin, Condenser capacities in geometric function theory, Dal'nevost. Univ., Vladivostok, 2003. (Russian)

[19] V. N. Dubinin and E. G. Prilepkina, Preservation of the generalized reduced module under some geometric transformations of the plane domains, Dal'nevost. Mat. Zh. 6 (2005), no. 1-2, 39-56. (Russian)

[20] P. Duren and M. Schiffer, Robin functions and energy functionals of multiply connected domains, Pacific J. Math. 148 (1991), 251-273. MR1094490 (92c:31003)

[21] L Robin functions and distortion of capacity under conformal mapping, Complex Variables Theory Appl. 21 (1993), 189-196. MR.1276575 (95g:30031)

[22] V. N. Dubinin and N. V. Ėrrikh, Some applications of generalized condensers in the theory of analytic functions, Zap. Nauchn. Sem. S.-Peterburg. Otdel. Mat. Inst. Steklov. (POMI) 314 (2004), 52-75; English transl., J. Math. Sci. (N. Y.) 133 (2006), no. 6, 1634-1647. MR2119734(2005i:30036)

[23] Ch. Pommerenke, Boundary behaviour of conformal maps, Grundlehren Math. Wiss., vol. 299, Springer-Verlag, Berlin, 1992. MR 1217706 (95b:30008)

[24] N. A. Lebedev, Some estimates for functions regular and univalent in a circle, Vestnik Leningrad. Univ. Ser. Mat. Fiz. Khim. 1955, vyp. 4, 3-21. (Russian) MR0074514 (17:599c)

[25] V. N. Dubinin, On the Schwarz inequality on the boundary for functions regular in the disk, Zap. Nauchn. Sem. S.-Peterburg. Otdel. Mat. Inst. Steklov. (POMI) 286 (2002), 74-84; English transl., J. Math. Sci. (New York) 122 (2004), no. 6, 3623-3629. MR1937369 (2003i:30036)

[26] _ Schwarz's lemma and coefficient estimates for regular functions with free definition domain, Mat. Sb. 196 (2005), no. 11, 53-74; English transl., Sb. Math. 196 (2005), no. 11-12, 1605-1625. MR2216010

[27] A. Yu. Solynin, Functional inequalities via polarization, Algebra i Analiz 8 (1996), no. 6, 148-185; English transl., St. Petersburg Math. J. 8 (1997), no. 6, 1015-1038. MR.1458141 (98e:30001a)

[28] D. Betsakos, Polarization, conformal invariants, and Brownian motion, Ann. Acad. Sci. Fenn. Math. 23 (1998), 59-82. MR1601843 (99g:31004)

[29] R. W. Barnard and A. Yu. Solynin, Local variations and minimal area problem for Carathéodory functions, Indiana Univ. Math. J. 53 (2004), 135-167. MR2048187(2005a:30028)

[30] R. W. Barnard, C. Richardson, and A. Yu. Solynin, Concentration of area in half-planes, Proc. Amer. Math. Soc. 133 (2005), 2091-2099. MR2137876 (2006b:30048)

[31] G. Pólya and G. Szegö, Isoperimetric inequalities in mathematical physics, Ann. of Math. Stud., no. 27, Princeton Univ. Press, Princeton, NJ, 1951. MR0043486 (13:270d)

[32] M. Marcus, Radial averaging of domains, estimates for Dirichlet integrals and applications, J. Anal. Math. 27 (1974), 47-78. MR0477029 (57:16573)

[33] V. N. Dubinin and M. Vuorinen, On conformal moduli of polygonal quadrilaterals, Preprint no. 417, Univ. Helsinki, Dep. Math. and Statist., 2005.

[34] A. W. Goodman, Univalent functions. Vols. I, II, Mariner Publ. Co., Inc., Tampa, FL, 1983. MR704183 (85j:30035a) MR0704184 (85j:30035b) 
[35] T. Kubo, Symmetrization and univalent functions in an annulus, J. Math. Soc. Japan 6 (1954), no. 1, 55-67. MR0062230 (15:948a)

[36] J. A. Jenkins, Univalent functions and conformal mapping, Ergeb. Math. Grenzgeb. (N. F.), no. 18, Springer-Verlag, Berlin, 1958. MR0096806 (20:3288)

[37] M. Ohtsuka, Dirichlet problem, extremal length and prime ends, New York, 1970.

[38] I. A. Aleksandrov, Parametric continuations in the theory of univalent functions, "Nauka", Moscow, 1976. (Russian) MR0480952 (58:1099)

[39] V. V. Goryaĭnov, Semigroups of conformal mapping, Mat. Sb. (N.S.) 129 (1986), no. 4, 451-472; English transl. in Math. USSR-Sb. 57 (1987), no. 2. MR842395 (87i:30018)

[40] I. A. Aleksandrov and B. G. Tsvetkov, Functions that confromally map the strip into itself, Sibirsk. Mat. Zh. 21 (1980), no. 1, 4-25; English transl., Siberian Math. J. 21 (1980), no. 1, 1-19. MR562016 (81e:30032)

Institute of Applied Mathematics, Far-East Branch, Russian Academy of Sciences, Ul. Radio 7, Vladivostok, 690041, Russia

E-mail address: dubinin@iam.dvo.ru

Institute of Applied Mathematics, Far-East Branch, Russian Academy of Sciences, Ul. Radio 7, Vladivostok, 690041, Russia

E-mail address: pril@mail.primorye.ru

Received 22/FEB/2006

Translated by S. V. KISLYAKOV 